\title{
Aktueller Überblick
}

\section{Grundlagen der T-Zell-vermittelten Ekzemreaktion}

Ekzemkrankheiten gehören zu den häufigsten Gründen, einen Dermatologen aufzusuchen. Insbesondere hat das atopische Ekzem in den letzten vier Jahrzehnten deutlich zugenommen. Während das allergische Kontaktekzem als Modellerkrankung für eine T-Zellvermittelte Überreaktion seit vielen Jahren etabliert ist, mehren sich die Hinweise, dass auch bei dem atopischen Ekzem spezifische, durch T-Zellen vermittelte Immunreaktionen gegen Nahrungsmittel und Aeroallergene bestehen.

A us diesem Grund besteht großes Interesse an Erkenntnissen über die Bedeutung spezifischer T-Lymphozyten bei diesem Krankheitsbild z.B. für die Entwicklung neuer diagnostischer Tests, wie dem Atopie-Patch-Test.

Der Autor des vorliegenden Buchs, Thomas Werfel, arbeitet seit vielen Jahren an der Aufklärung von T-Zellvermittelten Mechanismen bei Ekzemreaktionen. In diesem Band werden die Ergebnisse zahlreicher experimenteller Untersuchungen seiner Arbeitsgruppe dargestellt.

Der Schwerpunkt liegt dabei auf Untersuchungen der Nahrungsmittelund Inhalationsallergen-spezifischen Immunantwort beim atopischen Ekzem. Aufgrund der Vielzahl der vorgestellten Untersuchungen sind die Texte und zahlreichen Tabellen sehr knapp gehalten. Im Diskussionsteil werden diese Ergebnisse erläutert und der Bezug zur angewandten Allergologie erkennbar.
Es findet sich ein Überblick über den aktuellen Stand der Forschung auf diesem Gebiet mit einem sehr umfangreichen Literaturverzeichnis zur weiteren Information.

Fazit: Wer wissenschaftlich auf diesem Gebiet arbeiten möchte findet einen guten Einstieg in diese Thematik. Ein Experte der Materie gibt in diesem Band einen guten Einblick in die aktuellen Erkenntnisse der zellulären Immunantwort bei den häufigen Ekzemreaktionen.

D. Wessner, J. Ring

Werfel T. Allergenspezifische T-ZellAntwort bei Ekzemkrankheiten. Dustri-Verlag, München-Deisenhofen 2000; XII, 106 Seiten, 42 Abbildungen, 33 Tabellen, broschiert, 98,- DM, ISBN 3-87185-293-7. 